Original Research Paper

\title{
Kinematics of a Mechanism with a Triad
}

\author{
${ }^{1}$ Relly Victoria Virgil Petrescu, ${ }^{2}$ Raffaella Aversa, \\ ${ }^{3}$ Taher M. Abu-Lebdeh, ${ }^{2}$ Antonio Apicella and ${ }^{1}$ Florian Ion Tiberiu Petrescu \\ ${ }^{I}$ ARoTMM-IFToMM, Bucharest Polytechnic University, Bucharest, (CE), Romania \\ ${ }^{2}$ Department of Architecture and Industrial Design, Advanced Material Lab, \\ Second University of Naples, 81031 Aversa (CE), Italy \\ ${ }^{3}$ North Carolina A and T State University, USA
}

Article history

Received: 25-02-2018

Revised: 26-02-2018

Accepted: 01-03-2018

Corresponding Author: Florian Ion Tiberiu Petres ARoTMM-IFToMM, Bucharest Polytechnic University, Bucharest, (CE), Romania Email: scipub02@gmail.com

\begin{abstract}
The Assuric Structural Groups are the most well-known classification and modulation used in machine and machine theory, and even though other modular classifications adapted to robots have emerged today, they still remain a reference classification in industrial mechanics and engineering. Diada in the mechanics can be studied similarly to the diode in the electronics, the triad is studied in the theory of machine and robot mechanisms similar to triode (or transistor) study in electronics. Further, the theory of the mechanisms is studying: tetrad, pentad ... but it cannot go further than for a 12th-order structural group because the efficiency of mechanisms using such very large groups is very small and such a mechanism can be blocked in operation. If the similarity between the mechanisms and the electronics is correct up to the 5-6th class, the larger ones are of no use, the advantages of the electronics being that it can also function in the large or very large group with high yields, without blockages, which is why the integrated circuits and electronic chips were born. The present work is intended presenting a triad kinematics general used only with the kinematic couplings rotational (C5), because such approaches are rare in the area, although triad is a structured group Assuric often used. The calculation method presented is an analytical one.
\end{abstract}

Keywords: Mechanisms, Robots, Mechatronics, Structural Groups, Dyad, Triad, Kinematics, Triad Kinematics, An Analytical Method

\section{Introduction}

In machines, mechanisms and robots theory, structural or modular groups are often used to ease calculations of various mechanisms used on machines and industrial robots.

The most well-known structural classification in groups is the Assuric one which use dyads and triads...

A less studied Assuric group is the triad, which is why the present paper wants to present the analytical kinematics of this third-class structural group, determined by an original method.

Structural groups have been thoroughly analyzed in some specialized papers (Pelecudi, 1967; Antonescu, 2000; Comănescu et al., 2010), but not from an analytical point of view, but more structurally and graphically.

The Assuric Structural Groups are the most wellknown classification and modulation used in machine and machine theory, and even though other modular classifications adapted to robots have emerged today, they still remain a reference classification in industrial mechanics and engineering.

Dyad in the mechanics can be studied similarly to the diode in the electronics, the triad is studied in the theory of machine and robot mechanisms similar to triode (or transistor) study in electronics. Further, the theory of the mechanisms is studying: tetrad, pentad ... but it cannot go further than for a 12th-order structural group because the efficiency of mechanisms using such very large groups is very small and such a mechanism can be blocked in operation. If the similarity between the mechanisms and the electronics is correct up to the 5-6th class, the larger ones are of no use, the advantages of the electronics being that it can also function in the large or very large group with high yields, without blockages, which is why the integrated circuits and electronic chips were born. 
The present work is intended presenting a triad kinematics general used only with the kinematic couplings rotational (C5), because such approaches are rare in the area, although triad is a structured group Assuric often used. The calculation method presented is an analytical one (Pelecudi, 1967; Antonescu, 2000; Comănescu et al., 2010; Aversa et al., 2016a; 2016b; 2016c; 2016d; 2017a; 2017b; 2017c; 2017d; 2017e; Berto et al., 2016a; 2016b; 2016c; 2016d; Mirsayar et al., 2017; Cao et al., 2013; Dong et al., 2013; De Melo et al., 2012; Garcia et al., 2007; Garcia-Murillo et al., 2013; He et al., 2013; Lee, 2013; Lin et al., 2013; Liu et al., 2013; Padula and Perdereau, 2013; Perumaal and Jawahar, 2013; Petrescu and Petrescu, 1995a; 1995b; 1997a; 1997b; 1997c; 2000a; 2000b; 2002a; 2002b; 2003; 2005a; 2005b; 2005c; 2005d; 2005e, 2016a; 2016b; 2016c; 2016d; 2016e; 2013; 2012a; 2012b; 2011; Petrescu et al., 2009; 2016a-e; 2017a-ae; Petrescu and Calautit, 2016a-b; Reddy et al., 2012; Tabaković et al., 2013; Tang et al., 2013; Tong et al., 2013; Wang et al., 2013; Wen et al., 2012; Antonescu and Petrescu, 1985; 1989; Antonescu et al., 1985a; 1985b; 1986; 1987; 1988; 1994; 1997; 2000a; 2000b; 2001).

\section{Materials and Methods}

The kinematic scheme of a $6 \mathrm{R}$ triad can be seen in Fig. 1.

The kinematic equations of positions are written for two independent contours in the form of the system (1).

Although a system of four equations with four unknowns results, solving the system is more difficult because the equations are transcendental.

$$
\left\{\begin{array}{l}
\left\{\begin{array}{l}
x_{B}+l_{2} \cdot \cos \varphi_{2}=x_{C}+l_{3} \cdot \cos \varphi_{3}+g \cdot \cos \left(\varphi_{5}+\alpha\right) \\
y_{B}+l_{2} \cdot \sin \varphi_{2}=y_{C}+l_{3} \cdot \sin \varphi_{3}+g \cdot \sin \left(\varphi_{5}+\alpha\right)
\end{array}\right. \\
\left\{\begin{array}{l}
x_{C}+l_{3} \cdot \cos \varphi_{3}+e \cdot \cos \varphi_{5}=x_{D}+l_{4} \cdot \cos \varphi_{4} \\
y_{C}+l_{3} \cdot \sin \varphi_{3}+e \cdot \sin \varphi_{5}=y_{D}+l_{4} \cdot \sin \varphi_{4}
\end{array}\right.
\end{array}\right.
$$

Write the system (1) in form (2) and lift each equation to square, then add the first two and the last two to eliminate the two unknown $\left(\varphi_{2}\right.$ and $\left.\varphi_{4}\right)$. We obtain the new system (3) of two equations with two unknowns which are arranged successively in the forms (4), (5) and (6).

$$
\left\{\begin{array}{l}
\left\{\begin{array}{l}
l_{2} \cdot \cos \varphi_{2}=x_{C}-x_{B}+l_{3} \cdot \cos \varphi_{3}+\left.g \cdot \cos \left(\varphi_{5}+\alpha\right)\right|^{\wedge} 2 \\
l_{2} \cdot \sin \varphi_{2}=y_{C}-y_{B}+l_{3} \cdot \sin \varphi_{3}+\left.g \cdot \sin \left(\varphi_{5}+\alpha\right)\right|^{\wedge} 2
\end{array} \Rightarrow I\right. \\
\left\{\begin{array}{l}
l_{4} \cdot \cos \varphi_{4}=x_{C}-x_{D}+l_{3} \cdot \cos \varphi_{3}+\left.e \cdot \cos \varphi_{5}\right|^{\wedge} 2 \\
l_{4} \cdot \sin \varphi_{4}=y_{C}-y_{D}+l_{3} \cdot \sin \varphi_{3}+\left.e \cdot \sin \varphi_{5}\right|^{\wedge} 2
\end{array} \Rightarrow I I\right.
\end{array}\right.
$$

$$
\begin{aligned}
& \left\{\begin{array}{l}
I: \quad l_{2}^{2}=\left[\left(x_{C}-x_{B}\right)+l_{3} \cdot \cos \varphi_{3}+g \cdot \cos \left(\varphi_{5}+\alpha\right)\right]^{2}+ \\
+\left[\left(y_{C}-y_{B}\right)+l_{3} \cdot \sin \varphi_{3}+g \cdot \sin \left(\varphi_{5}+\alpha\right)\right]^{2} \\
I I: \quad l_{4}^{2}=\left[\left(x_{C}-x_{D}\right)+l_{3} \cdot \cos \varphi_{3}+e \cdot \cos \varphi_{5}\right]^{2}+ \\
+\left[\left(y_{C}-y_{D}\right)+l_{3} \cdot \sin \varphi_{3}+e \cdot \sin \varphi_{5}\right]^{2}
\end{array}\right. \\
& l_{2}^{2}=\left(x_{C}-x_{B}\right)^{2}+\left(y_{C}-y_{B}\right)^{2}+l_{3}^{2}+g^{2}+ \\
& 2 \cdot l_{3} \cdot\left(x_{C}-x_{B}\right) \cdot \cos \varphi_{3}+2 \cdot l_{3} \cdot\left(y_{C}-y_{B}\right) \cdot \sin \varphi_{3} \\
& +2 \cdot g \cdot\left(x_{C}-x_{B}\right) \cdot \cos \left(\varphi_{5}+\alpha\right)+2 \cdot g \cdot\left(y_{C}-y_{B}\right) \text {. } \\
& \sin \left(\varphi_{5}+\alpha\right)+2 \cdot g \cdot l_{3} \cdot \cos \left(\varphi_{5}+\alpha-\varphi_{3}\right) \\
& l_{4}^{2}=\left(x_{C}-x_{D}\right)^{2}+\left(y_{C}-y_{D}\right)^{2}+l_{3}^{2}+e^{2}+ \\
& 2 \cdot l_{3} \cdot\left(x_{C}-x_{D}\right) \cdot \cos \varphi_{3}+2 \cdot l_{3} \cdot\left(y_{C}-y_{D}\right) \cdot \sin \varphi_{3} \\
& +2 \cdot e \cdot\left(x_{C}-x_{D}\right) \cdot \cos \varphi_{5}+ \\
& +2 \cdot e \cdot\left(y_{C}-y_{D}\right) \cdot \sin \varphi_{5}+2 \cdot e \cdot l_{3} \cdot \cos \left(\varphi_{5}-\varphi_{3}\right) \\
& \left\{\begin{array}{l}
l_{2}^{2}=\left(x_{C}-x_{B}\right)^{2}+\left(y_{C}-y_{B}\right)^{2}+l_{3}^{2}+g^{2}+ \\
2 \cdot l_{3} \cdot\left(x_{C}-x_{B}\right) \cdot \cos \varphi_{3}+2 \cdot l_{3} \cdot\left(y_{C}-y_{B}\right) \cdot \sin \varphi_{3} \\
+2 \cdot g \cdot\left(x_{C}-x_{B}\right) \cdot \cos \alpha \cdot \cos \varphi_{5}- \\
-2 \cdot g \cdot\left(x_{C}-x_{B}\right) \cdot \sin \alpha \cdot \sin \varphi_{5}+ \\
2 \cdot g \cdot\left(y_{C}-y_{B}\right) \cdot \sin \alpha \cdot \cos \varphi_{5}+ \\
2 \cdot g \cdot\left(y_{C}-y_{B}\right) \cdot \cos \alpha \cdot \sin \varphi_{5}+ \\
2 \cdot g \cdot l_{3} \cdot \cos \alpha \cdot \cos \left(\varphi_{5}-\varphi_{3}\right)- \\
2 \cdot g \cdot l_{3} \cdot \sin \alpha \cdot \sin \left(\varphi_{5}-\varphi_{3}\right) \\
l_{4}^{2}=\left(x_{C}-x_{D}\right)^{2}+\left(y_{C}-y_{D}\right)^{2}+l_{3}^{2}+e^{2}+ \\
2 \cdot l_{3} \cdot\left(x_{C}-x_{D}\right) \cdot \cos \varphi_{3}+2 \cdot l_{3} \cdot\left(y_{C}-y_{D}\right) \cdot \sin \varphi_{3} \\
+2 \cdot e \cdot\left(x_{C}-x_{D}\right) \cdot \cos \varphi_{5}+ \\
+2 \cdot e \cdot\left(y_{C}-y_{D}\right) \cdot \sin \varphi_{5}+2 \cdot e \cdot l_{3} \cdot \cos \left(\varphi_{5}-\varphi_{3}\right)
\end{array}\right. \\
& \left\{\begin{array}{l}
l_{2}^{2}=\left(x_{C}-x_{B}\right)^{2}+\left(y_{C}-y_{B}\right)^{2}+l_{3}^{2}+g^{2}+ \\
2 \cdot l_{3} \cdot\left(x_{C}-x_{B}\right) \cdot \cos \varphi_{3}+2 \cdot l_{3} \cdot\left(y_{C}-y_{B}\right) \cdot \sin \varphi_{3}+ \\
2 \cdot g \cdot\left[\left(x_{C}-x_{B}\right) \cdot \cos \alpha+\left(y_{C}-y_{B}\right) \cdot \sin \alpha\right] \cdot \cos \varphi_{5}+ \\
2 \cdot g \cdot\left[\left(y_{C}-y_{B}\right) \cdot \cos \alpha-\left(x_{C}-x_{B}\right) \cdot \sin \alpha\right] \cdot \sin \varphi_{5}+ \\
2 \cdot g \cdot l_{3} \cdot \cos \alpha \cdot \cos \left(\varphi_{5}-\varphi_{3}\right)- \\
2 \cdot g \cdot l_{3} \cdot \sin \alpha \cdot \sin \left(\varphi_{5}-\varphi_{3}\right) \\
l_{4}^{2}=\left(x_{C}-x_{D}\right)^{2}+\left(y_{C}-y_{D}\right)^{2}+l_{3}^{2}+e^{2}+ \\
2 \cdot l_{3} \cdot\left(x_{C}-x_{D}\right) \cdot \cos \varphi_{3}+2 \cdot l_{3} \cdot\left(y_{C}-y_{D}\right) \cdot \sin \varphi_{3} \\
+2 \cdot e \cdot\left(x_{C}-x_{D}\right) \cdot \cos \varphi_{5}+ \\
+2 \cdot e \cdot\left(y_{C}-y_{D}\right) \cdot \sin \varphi_{5}+2 \cdot e \cdot l_{3} \cdot \cos \left(\varphi_{5}-\varphi_{3}\right)
\end{array}\right.
\end{aligned}
$$


In order to solve the transcedental system (6), the method of successive approximations is used, considering the known trigonometric functions by knowing the angles $\varphi_{3}$ and $\varphi_{5}$ (they are given an initial value of any of these two angles for the priming of the iterative calculations) and the differences are calculated $\Delta \varphi_{3}$ and $\Delta \varphi_{5}$. The system (6) is rewritten into shape (8) by replacing the angles with the angle plus a difference according to the relations (7).

$$
\left\{\begin{array}{l}
\left\{\begin{array}{l}
\cos \varphi_{3} \Rightarrow \cos \varphi_{3}-\Delta \varphi_{3} \cdot \sin \varphi_{3} \\
\sin \varphi_{3} \Rightarrow \sin \varphi_{3}+\Delta \varphi_{3} \cdot \cos \varphi_{3} \\
\cos \varphi_{5} \Rightarrow \cos \varphi_{5}-\Delta \varphi_{5} \cdot \sin \varphi_{5} \\
\sin \varphi_{5} \Rightarrow \sin \varphi_{5}+\Delta \varphi_{5} \cdot \cos \varphi_{5} \\
\cos \left(\varphi_{5}-\varphi_{3}\right) \Rightarrow \\
\cos \left(\varphi_{5}-\varphi_{3}\right)-\left(\Delta \varphi_{5}-\Delta \varphi_{3}\right) \cdot \sin \left(\varphi_{5}-\varphi_{3}\right) \\
\sin \left(\varphi_{5}-\varphi_{3}\right) \Rightarrow \\
\sin \left(\varphi_{5}-\varphi_{3}\right)+\left(\Delta \varphi_{5}-\Delta \varphi_{3}\right) \cdot \cos \left(\varphi_{5}-\varphi_{3}\right)
\end{array}\right. \\
\left\{\begin{array}{l}
l_{2}^{2}=\left(x_{C}-x_{B}\right)^{2}+\left(y_{C}-y_{B}\right)^{2}+l_{3}^{2}+g^{2}+ \\
2 \cdot l_{3} \cdot\left(x_{C}-x_{B}\right) \cdot \cos \varphi_{3}+2 \cdot l_{3} \cdot\left(y_{C}-y_{B}\right) \cdot \sin \varphi_{3} \\
-2 \cdot l_{3} \cdot\left(x_{C}-x_{B}\right) \cdot \sin \varphi_{3} \cdot \Delta \varphi_{3}+ \\
+2 \cdot l_{3} \cdot\left(y_{C}-y_{B}\right) \cdot \cos \varphi_{3} \cdot \Delta \varphi_{3}+ \\
+2 \cdot g \cdot\left[\left(x_{C}-x_{B}\right) \cdot \cos \alpha+\left(y_{C}-y_{B}\right) \cdot \sin \alpha\right] \cdot \cos \varphi_{5} \\
+2 \cdot g \cdot\left[\left(x_{C}-x_{B}\right) \cdot \cos \alpha+\left(y_{C}-y_{B}\right) \cdot \sin \alpha\right] \cdot \sin \varphi_{5} \cdot \Delta \varphi_{5} \\
+2 \cdot e \cdot l_{3} \cdot \cos \left(\varphi_{5}-\varphi_{3}\right)-2 \cdot e \cdot l_{3} \cdot \sin \left(\varphi_{5}-\varphi_{3}\right) \cdot \Delta \varphi_{5} \\
+2 \cdot \sin \left(\varphi_{5}-\varphi_{3}\right) \cdot \Delta \varphi_{3} \\
+2 \cdot g \cdot\left[\left(y_{C}-y_{B}\right) \cdot \cos \alpha-\left(x_{C}-x_{B}\right) \cdot \sin \alpha\right] \cdot \sin \varphi_{5} \\
+2 \cdot l_{3} \cdot\left(x_{C}-x_{D}\right) \cdot \cos \varphi_{3}-2 \cdot l_{3} \cdot\left(x_{C}-x_{D}\right) \cdot \sin \varphi_{3}+2 \cdot l_{3} \cdot\left(y_{C}-y_{D}\right) \cdot \sin \varphi_{3} \cdot \Delta \varphi_{3} \\
+2 \cdot g \cdot\left[\left(y_{C}-y_{B}\right) \cdot \cos \alpha-\left(x_{C}-x_{B}\right) \cdot \sin \alpha\right] \cdot \cos \varphi_{5} \cdot \Delta \varphi_{5} \\
+2 \cdot g \cdot l_{3} \cdot \cos \alpha \cdot \cos \left(\varphi_{5}-\varphi_{3}\right)- \\
-2 \cdot g \cdot l_{3} \cdot \cos \alpha \cdot \sin \left(\varphi_{5}-\varphi_{3}\right) \cdot \Delta \varphi_{5}+ \\
+2 \cdot g \cdot l_{3} \cdot \cos \alpha \cdot \sin \left(\varphi_{5}-\varphi_{3}\right) \cdot \Delta \varphi_{3}- \\
-2 \cdot g \cdot l_{3} \cdot \sin \alpha \cdot \sin \left(\varphi_{5}-\varphi_{3}\right)- \\
-2 \cdot g \cdot l_{3} \cdot \sin \alpha \cdot \cos \left(\varphi_{5}-\varphi_{3}\right) \cdot \Delta \varphi_{5}+ \\
+2 \cdot g \cdot l_{3} \cdot \sin \alpha \cdot \cos \left(\varphi_{5}-\varphi_{3}\right) \cdot \Delta \varphi_{3}
\end{array}\right.
\end{array}\right.
$$

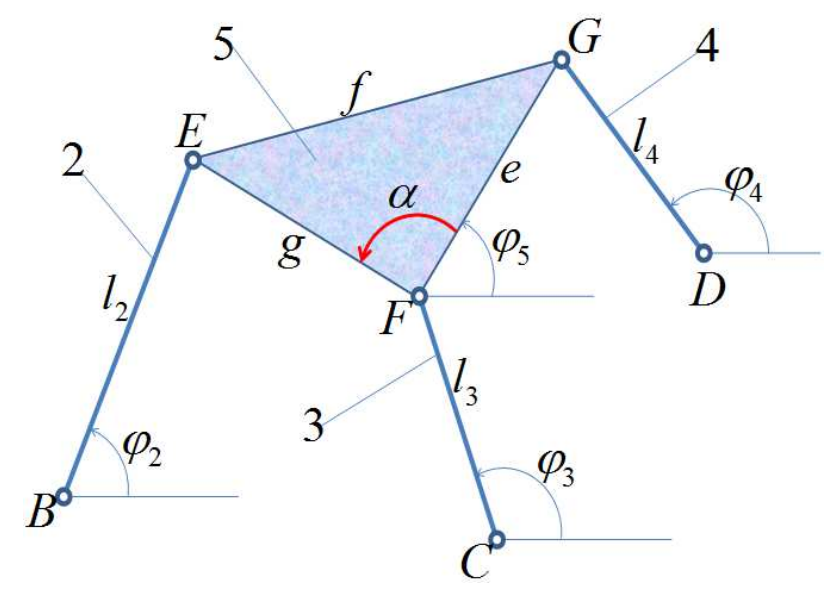

Fig. 1: The kinematic scheme of a $6 \mathrm{R}$ triad

The system (8) is arranged in the form (9) by grouping the corresponding terms so that a linear system of two equations with two unknowns appears, the unknown being $\Delta \varphi_{3}$ and $\Delta \varphi_{5}$.

It is now clear what the purpose of adding finite differences was. The nonlinear system has linearized in the form of a type system (10).

The solutions of the system (10) are given by the relations (11).

$$
\left\{\begin{array}{l}
\left\{2 \cdot l_{3} \cdot\left[\left(y_{C}-y_{B}\right) \cdot \cos \varphi_{3}-\left(x_{C}-x_{B}\right) \cdot \sin \varphi_{3}\right]\right. \\
\left.+2 \cdot g \cdot l_{3} \cdot \sin \left(\varphi_{5}-\varphi_{3}+\alpha\right)\right\} \cdot \Delta \varphi_{3} \\
2 \cdot g \cdot\left\{\left(y_{C}-y_{B}\right) \cdot \cos \alpha \cdot \cos \varphi_{5}\right. \\
-\left(x_{C}-x_{B}\right) \cdot \sin \alpha \cdot \cos \varphi_{5}-\left(x_{C}-x_{B}\right) \cdot \cos \alpha \cdot \sin \varphi_{5} \\
-\left(y_{C}-y_{B}\right) \cdot \sin \alpha \cdot \sin \varphi_{5}-l_{3} \cdot\left[\cos \alpha \cdot \sin \left(\varphi_{5}-\varphi_{3}\right)\right. \\
\left.\left.+\sin \alpha \cdot \cos \left(\varphi_{5}-\varphi_{3}\right)\right]\right\} \cdot \Delta \varphi_{5}= \\
=l_{2}^{2}-\left(x_{C}-x_{B}\right)^{2}-\left(y_{C}-y_{B}\right)^{2}-l_{3}^{2}-g^{2} \\
-2 \cdot l_{3} \cdot\left(x_{C}-x_{B}\right) \cdot \cos \varphi_{3}-2 \cdot l_{3} \cdot\left(y_{C}-y_{B}\right) \cdot \sin \varphi_{3} \\
-2 \cdot g \cdot\left\{\left[\left(x_{C}-x_{B}\right) \cdot \cos \alpha+\left(y_{C}-y_{B}\right) \cdot \sin \alpha\right] \cdot \cos \varphi_{5}\right. \\
\left.+\left[\left(y_{C}-y_{B}\right) \cdot \cos \alpha-\left(x_{C}-x_{B}\right) \cdot \sin \alpha\right] \cdot \sin \varphi_{5}\right\}- \\
-2 \cdot g \cdot l_{3} \cdot\left[\cos \alpha \cdot \cos \left(\varphi_{5}-\varphi_{3}\right)-\sin \alpha \cdot \sin \left(\varphi_{5}-\varphi_{3}\right)\right] \\
\\
2 l_{3} \cdot\left[\left(y_{C}-y_{D}\right) \cos \varphi_{3}-\left(x_{C}-x_{D}\right) \sin \varphi_{3}+e \sin \left(\varphi_{5}-\varphi_{3}\right)\right] \Delta \varphi_{3} \\
+2 e \cdot\left[\left(y_{C}-y_{D}\right) \cos \varphi_{5}-\left(x_{C}-x_{D}\right) \sin \varphi_{5}-l_{3} \sin \left(\varphi_{5}-\varphi_{3}\right)\right] \Delta \varphi_{5} \\
=l_{4}^{2}-\left(x_{C}-x_{D}\right)^{2}-\left(y_{C}-y_{D}\right)^{2}-l_{3}^{2}-e^{2} \\
-2 \cdot l_{3} \cdot\left(x_{C}-x_{D}\right) \cdot \cos \varphi_{3}-2 \cdot l_{3} \cdot\left(y_{C}-y_{D}\right) \cdot \sin \varphi_{3} \\
-2 \cdot e \cdot\left(x_{C}-x_{D}\right) \cdot \cos \varphi_{5}-2 \cdot e \cdot\left(y_{C}-y_{D}\right) \cdot \sin \varphi_{5} \\
-2 \cdot e \cdot l_{3} \cdot \cos \left(\varphi_{5}-\varphi_{3}\right)
\end{array}\right.
$$




$$
\left\{\begin{array}{l}
a_{11} \cdot \Delta \varphi_{3}+a_{12} \cdot \Delta \varphi_{5}=a_{1} \\
a_{21} \cdot \Delta \varphi_{3}+a_{22} \cdot \Delta \varphi_{5}=a_{2}
\end{array}\right.
$$

$$
\left\{\begin{array}{l}
\Delta=\left|\begin{array}{ll}
a_{11} & a_{12} \\
a_{21} & a_{22}
\end{array}\right|=a_{11} a_{22}-a_{12} a_{21} ; \\
\Delta_{3}=\left|\begin{array}{ll}
a_{1} & a_{12} \\
a_{2} & a_{22}
\end{array}\right|=a_{1} \cdot a_{22}-a_{12} \cdot a_{2} \\
\Delta_{5}=\left|\begin{array}{ll}
a_{11} & a_{1} \\
a_{21} & a_{2}
\end{array}\right|=a_{11} \cdot a_{2}-a_{1} \cdot a_{21} \\
\Rightarrow \Delta \varphi_{3}=\frac{\Delta_{3}}{\Delta} ; \Delta \varphi_{5}=\frac{\Delta_{5}}{\Delta}
\end{array}\right.
$$

The coefficients of the system (10) are identified by (9) given by the relational system (12).

$$
\left\{\begin{array}{l}
a_{11}=2 \cdot l_{3} \cdot\left[\left(y_{C}-y_{B}\right) \cdot \cos \varphi_{3}-\left(x_{C}-x_{B}\right) \cdot \sin \varphi_{3}\right. \\
\left.+g \cdot \sin \left(\varphi_{5}-\varphi_{3}+\alpha\right)\right] \\
a_{12}=2 \cdot g \cdot\left[\left(y_{C}-y_{B}\right) \cdot \cos \left(\varphi_{5}+\alpha\right)-\right. \\
\left.\left(x_{C}-x_{B}\right) \sin \left(\varphi_{5}+\alpha\right)-l_{3} \cdot \sin \left(\varphi_{5}-\varphi_{3}+\alpha\right)\right] \\
a_{1}=l_{2}^{2}-l_{3}^{2}-g^{2}-\left(x_{C}-x_{B}\right)^{2}-\left(y_{C}-y_{B}\right)^{2}- \\
-2 \cdot l_{3} \cdot\left[\left(x_{C}-x_{B}\right) \cdot \cos \varphi_{3}+\left(y_{C}-y_{B}\right) \cdot \sin \varphi_{3}\right]- \\
-2 \cdot g \cdot\left[\left(x_{C}-x_{B}\right) \cdot \cos \left(\varphi_{5}+\alpha\right)+\left(y_{C}-y_{B}\right) \cdot\right. \\
\left.\sin \left(\varphi_{5}+\alpha\right)+l_{3} \cdot \cos \left(\varphi_{5}-\varphi_{3}+\alpha\right)\right] \\
a_{21}=2 \cdot l_{3} \cdot \\
{\left[\left(y_{C}-y_{D}\right) \cos \varphi_{3}-\left(x_{C}-x_{D}\right) \sin \varphi_{3}+e \sin \left(\varphi_{5}-\varphi_{3}\right)\right]} \\
a_{22}=2 \cdot e \cdot \\
{\left[\left(y_{C}-y_{D}\right) \cos \varphi_{5}-\left(x_{C}-x_{D}\right) \sin \varphi_{5}-l_{3} \sin \left(\varphi_{5}-\varphi_{3}\right)\right]} \\
a_{2}=l_{4}^{2}-l_{3}^{2}-e^{2}-\left(x_{C}-x_{D}\right)^{2}-\left(y_{C}-y_{D}\right)^{2}- \\
-2 \cdot l_{3} \cdot\left(x_{C}-x_{D}\right) \cdot \cos \varphi_{3}-2 \cdot l_{3} \cdot\left(y_{C}-y_{D}\right) \cdot \sin \varphi_{3}- \\
-2 \cdot e \cdot\left(x_{C}-x_{D}\right) \cdot \cos \varphi_{5}-2 \cdot e \cdot\left(y_{C}-y_{D}\right) \cdot \sin \varphi_{5}- \\
-2 \cdot e \cdot l_{3} \cdot \cos \left(\varphi_{5}-\varphi_{3}\right) \\
\end{array}\right.
$$

In step 1 it is determined $\Delta \varphi \Delta \phi_{3}^{0}$ and $\Delta \phi_{5}^{0}$ in radii, which are added to the values initially considered to obtain the angular values for the first iteration, according to the system (13).

$$
\left\{\begin{array}{l}
\varphi_{3}^{1}=\varphi_{3}^{0}+\Delta \varphi_{3}^{0} \\
\varphi_{5}^{1}=\varphi_{5}^{0}+\Delta \varphi_{5}^{0}
\end{array}\right.
$$

If the values obtained are very close to the exact ones, the iterative process stops. Otherwise successive approximations will continue until the desired values are obtained. The final values $\varphi_{3}$ and $\varphi_{5}$ are considered to be $\mathrm{OK}$ when the error (difference) from their calculated value at the previous step is small enough.

It then returns to the initial positional systems to determine the other two values, $\varphi_{2}$ and $\varphi_{4}$ using the system (14):

$$
\begin{aligned}
& \iint \cos \varphi_{2}=\frac{x_{C}-x_{B}+l_{3} \cdot \cos \varphi_{3}+g \cdot \cos \left(\varphi_{5}+\alpha\right)}{l_{2}} \\
& \sin \varphi_{2}=\frac{y_{C}-y_{B}+l_{3} \cdot \sin \varphi_{3}+g \cdot \sin \left(\varphi_{5}+\alpha\right)}{l_{2}} \\
& \Rightarrow \varphi_{2} \\
& \varphi_{2}=\operatorname{semn}\left(\sin \varphi_{2}\right) \cdot \arccos \left(\cos \varphi_{2}\right) \\
& \int \cos \varphi_{4}=\frac{x_{C}-x_{D}+l_{3} \cdot \cos \varphi_{3}+e \cdot \cos \varphi_{5}}{l_{4}} \\
& \sin \varphi_{4}=\frac{y_{C}-y_{D}+l_{3} \cdot \sin \varphi_{3}+e \cdot \sin \varphi_{5}}{l_{4}} \\
& \Rightarrow \varphi_{4} \\
& \varphi_{4}=\operatorname{semn}\left(\sin \varphi_{4}\right) \cdot \arccos \left(\cos \varphi_{4}\right)
\end{aligned}
$$

Once the four angular positions have been determined, the initial systems are derived to obtain angular velocities and then angular accelerations.

The positioning system (1) is first derived to obtain the linear speed system (15):

$$
\left\{\begin{array}{l}
\left\{\begin{array}{l}
\dot{x}_{B}-l_{2} \cdot \sin \varphi_{2} \cdot \omega_{2}= \\
\dot{x}_{C}-l_{3} \cdot \sin \varphi_{3} \cdot \omega_{3}-g \cdot \sin \left(\varphi_{5}+\alpha\right) \cdot \omega_{5} \\
\dot{y}_{B}+l_{2} \cdot \cos \varphi_{2} \cdot \omega_{2}= \\
\dot{y}_{C}+l_{3} \cdot \cos \varphi_{3} \cdot \omega_{3}+g \cdot \cos \left(\varphi_{5}+\alpha\right) \cdot \omega_{5}
\end{array}\right. \\
\left\{\begin{array}{l}
\dot{x}_{C}-l_{3} \cdot \sin \varphi_{3} \cdot \omega_{3}-e \cdot \sin \varphi_{5} \cdot \omega_{5}= \\
\dot{x}_{D}-l_{4} \cdot \sin \varphi_{4} \cdot \omega_{4} \\
\dot{y}_{C}+l_{3} \cdot \cos \varphi_{3} \cdot \omega_{3}+e \cdot \cos \varphi_{5} \cdot \omega_{5}= \\
\dot{y}_{D}+l_{4} \cdot \cos \varphi_{4} \cdot \omega_{4}
\end{array}\right.
\end{array}\right.
$$


For the simpler solution of the system (15) we eliminate in the first phase two of the four unknown by multiplying the first equation of the system with $\cos \varphi_{2}$, the second with $\sin \varphi_{2}$, the third with $\cos \varphi_{4}$ and the last with $\sin \varphi_{4}$. Then the first two equations and the last two are collected, resulting in the system (16) formed by two linear equations with two unknown equations:

$$
\left\{\begin{array}{l}
\left(\dot{x}_{B}-\dot{x}_{C}\right) \cdot \cos \varphi_{2}+\left(\dot{y}_{B}-\dot{y}_{C}\right) \cdot \sin \varphi_{2}= \\
=l_{3} \cdot \sin \left(\varphi_{2}-\varphi_{3}\right) \cdot \omega_{3}+g \cdot \sin \left(\varphi_{2}-\varphi_{5}-\alpha\right) \cdot \omega_{5} \\
\left(\dot{x}_{D}-\dot{x}_{C}\right) \cdot \cos \varphi_{4}+\left(\dot{y}_{D}-\dot{y}_{C}\right) \cdot \sin \varphi_{4}= \\
=l_{3} \cdot \sin \left(\varphi_{4}-\varphi_{3}\right) \cdot \omega_{3}+e \cdot \sin \left(\varphi_{4}-\varphi_{5}\right) \cdot \omega_{5}
\end{array}\right.
$$

To solve the system (16) we apply two steps.

In the first step, the first system equation is amplified with $e \cdot \sin \left(\varphi_{4}-\varphi_{5}\right)$ and the second with $-g$. $\sin \left(\varphi_{2}-\varphi_{5}-\alpha\right)$.

We then gather the two expressions obtained and result a relationship from which we explicitly explain it $\omega_{3}$ (see expression 17):

$$
\left\{\begin{array}{l}
\omega_{3}=\left[e \cdot\left(\left(\dot{x}_{B}-\dot{x}_{C}\right) \cdot \cos \varphi_{2}+\left(\dot{y}_{B}-\dot{y}_{C}\right) \cdot \sin \varphi_{2}\right) .\right. \\
\sin \left(\varphi_{4}-\varphi_{5}\right)-g \cdot\left(\left(\dot{x}_{D}-\dot{x}_{C}\right) \cdot \cos \varphi_{4}+\left(\dot{y}_{D}-\dot{y}_{C}\right) .\right. \\
\left.\left.\sin \varphi_{4}\right) \cdot \sin \left(\varphi_{2}-\varphi_{5}-\alpha\right)\right] /\left[l_{3} .\right. \\
\left(e \sin \left(\varphi_{2}-\varphi_{3}\right) \sin \left(\varphi_{4}-\varphi_{5}\right)-\right. \\
\left.\left.g \sin \left(\varphi_{4}-\varphi_{3}\right) \sin \left(\varphi_{2}-\varphi_{5}-\alpha\right)\right)\right]
\end{array}\right.
$$

In the second step, the first system equation is amplified with $\sin \left(\varphi_{4}-\varphi_{3}\right)$ and the second with $-\sin \left(\varphi_{2}-\varphi_{3}\right)$.

We then gather the two expressions obtained and result a relationship from which we explicitly explain it $\omega_{5}$ (see expression 18 ):

$$
\begin{aligned}
& \omega_{5}=\left\{\left[\left(\dot{x}_{B}-\dot{x}_{C}\right) \cdot \cos \varphi_{2}+\left(\dot{y}_{B}-\dot{y}_{C}\right) \cdot \sin \varphi_{2}\right] \cdot \sin \left(\varphi_{4}-\varphi_{3}\right)-\right. \\
& \left.-\left[\left(\dot{x}_{D}-\dot{x}_{C}\right) \cdot \cos \varphi_{4}+\left(\dot{y}_{D}-\dot{y}_{C}\right) \cdot \sin \varphi_{4}\right] \cdot \sin \left(\varphi_{2}-\varphi_{3}\right)\right\} / \\
& {\left[g \sin \left(\varphi_{4}-\varphi_{3}\right) \sin \left(\varphi_{2}-\varphi_{5}-\alpha\right)-e \sin \left(\varphi_{2}-\varphi_{3}\right) \sin \left(\varphi_{4}-\varphi_{5}\right)\right]}
\end{aligned}
$$

From the system (15) it is then explained from the first two equations amplified with $-\sin \varphi_{2}$ respectively $\cos \varphi_{2}$ the angular velocity $\omega_{2}$, (relation 19), and from the last two relations amplified with $-\sin \varphi_{4}$ respectively $\cos \varphi_{4}$ the angular velocity, $\omega_{4}$ (relation 20 ):

$$
\begin{aligned}
& \omega_{2}=\frac{\left(\dot{x}_{B}-\dot{x}_{C}\right) \cdot \sin \varphi_{2}+\left(\dot{y}_{C}-\dot{y}_{B}\right) \cdot \cos \varphi_{2}}{l_{2}}+ \\
& +\frac{l_{3} \cdot \omega_{3} \cdot \cos \left(\varphi_{3}-\varphi_{2}\right)+g \cdot \omega_{5} \cdot \cos \left(\varphi_{2}-\varphi_{5}-\alpha\right)}{l_{2}}
\end{aligned}
$$

$$
\begin{aligned}
& \omega_{4}=\frac{\left(\dot{x}_{D}-\dot{x}_{C}\right) \cdot \sin \varphi_{4}+\left(\dot{y}_{C}-\dot{y}_{D}\right) \cdot \cos \varphi_{4}}{l_{4}}+ \\
& +\frac{l_{3} \cdot \omega_{3} \cdot \cos \left(\varphi_{4}-\varphi_{3}\right)+e \cdot \omega_{5} \cdot \cos \left(\varphi_{4}-\varphi_{5}\right)}{l_{4}}
\end{aligned}
$$

Appropriate angular accelerations are obtained most safely by direct derivation of corresponding angular velocities expressions.

Write the expression (17) deployed (in form 21) to make it easier to derive.

$$
\left\{\begin{array}{l}
\omega_{3} l_{3}\left[e \sin \left(\varphi_{2}-\varphi_{3}\right) \sin \left(\varphi_{4}-\varphi_{5}\right)-\right. \\
\left.g \sin \left(\varphi_{4}-\varphi_{3}\right) \sin \left(\varphi_{2}-\varphi_{5}-\alpha\right)\right] \\
=e \cdot\left[\left(\dot{x}_{B}-\dot{x}_{C}\right) \cdot \cos \varphi_{2}+\left(\dot{y}_{B}-\dot{y}_{C}\right) \cdot \sin \varphi_{2}\right] \\
\sin \left(\varphi_{4}-\varphi_{5}\right)- \\
-g \cdot\left[\left(\dot{x}_{D}-\dot{x}_{C}\right) \cdot \cos \varphi_{4}+\left(\dot{y}_{D}-\dot{y}_{C}\right) \cdot \sin \varphi_{4}\right] \\
\sin \left(\varphi_{2}-\varphi_{5}-\alpha\right)
\end{array}\right.
$$

The expression (21) of the angular velocity $\omega_{3}$ in relation to time is directly derived, and the corresponding angular acceleration (22) expression is obtained $\varepsilon_{3}$, which then immediately becomes the form (23):

$$
\left\{\begin{array}{l}
\varepsilon_{3} l_{3}\left[e \sin \left(\varphi_{2}-\varphi_{3}\right) \sin \left(\varphi_{4}-\varphi_{5}\right)-\right. \\
\left.g \sin \left(\varphi_{4}-\varphi_{3}\right) \sin \left(\varphi_{2}-\varphi_{5}-\alpha\right)\right] \\
=-\omega_{3} \cdot\left[l_{3} \cdot e \cdot \cos \left(\varphi_{2}-\varphi_{3}\right) \cdot \sin \left(\varphi_{4}-\varphi_{5}\right)\right. \\
\cdot\left(\omega_{2}-\omega_{3}\right)+l_{3} \cdot e \cdot \sin \left(\varphi_{2}-\varphi_{3}\right) \cdot \cos \left(\varphi_{4}-\varphi_{5}\right) \cdot \\
\left(\omega_{4}-\omega_{5}\right)- \\
-l_{3} \cdot g \cdot \cos \left(\varphi_{4}-\varphi_{3}\right) \cdot \sin \left(\varphi_{2}-\varphi_{5}-\alpha\right) \cdot\left(\omega_{4}-\omega_{3}\right)- \\
\left.-l_{3} \cdot g \cdot \sin \left(\varphi_{4}-\varphi_{3}\right) \cdot \cos \left(\varphi_{2}-\varphi_{5}-\alpha\right) \cdot\left(\omega_{2}-\omega_{5}\right)\right]+ \\
+e \cdot\left[\left(\ddot{x}_{B}-\ddot{x}_{C}\right) \cdot \cos \varphi_{2}+\left(\ddot{y}_{B}-\ddot{y}_{C}\right) \cdot \sin \varphi_{2}-\right. \\
\left.-\left(\dot{x}_{B}-\dot{x}_{C}\right) \cdot \sin \varphi_{2} \cdot \omega_{2}+\left(\dot{y}_{B}-\dot{y}_{C}\right) \cdot \cos \varphi_{2} \cdot \omega_{2}\right] \\
\sin \left(\varphi_{4}-\varphi_{5}\right)+e\left[\left(\dot{x}_{B}-\dot{x}_{C}\right) \cos \varphi_{2}+\left(\dot{y}_{B}-\dot{y}_{C}\right) \sin \varphi_{2}\right] \\
\cdot \cos \left(\phi_{4}-\phi_{5}\right)\left(\omega_{4}-\omega_{5}\right)- \\
-g \cdot\left[\left(\ddot{x}_{D}-\ddot{x}_{C}\right) \cdot \cos \varphi_{4}+\left(\ddot{y}_{D}-\ddot{y}_{C}\right) \cdot \sin \varphi_{4}-\right. \\
\left.-\left(\dot{x}_{D}-\dot{x}_{C}\right) \sin \varphi_{4} \cdot \omega_{4}+\left(\dot{y}_{D}-\dot{y}_{C}\right) \cos \varphi_{4} \cdot \omega_{4}\right] \\
\cdot \sin \left(\varphi_{2}-\varphi_{5}-\alpha\right)- \\
g\left[\left(\dot{x}_{D}-\dot{x}_{C}\right) \cos \varphi_{4}+\left(\dot{y}_{D}-\dot{y}_{C}\right) \sin \varphi_{4}\right] \\
\cdot \cos \left(\varphi_{2}-\varphi_{5}-\alpha\right)\left(\omega_{2}-\omega_{5}\right)
\end{array}\right.
$$




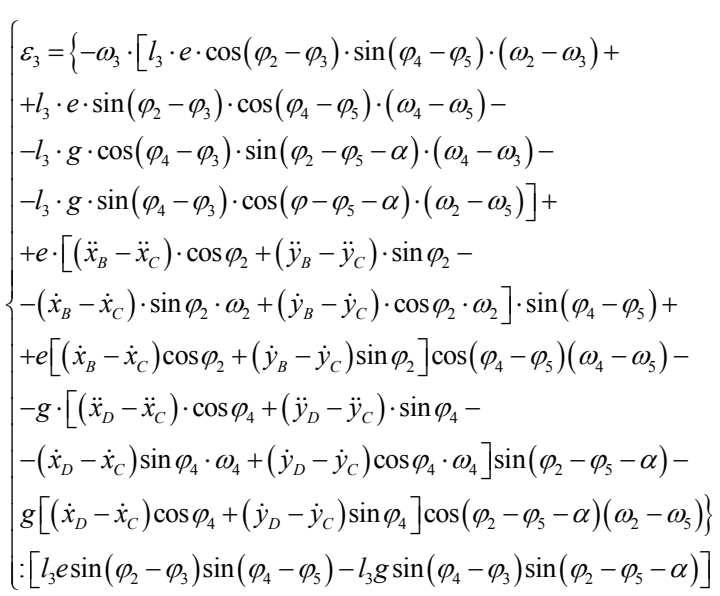

The angular velocity $\omega_{5}$ (relationship 24) is then written so that it can easily be derived:

$$
\left\{\begin{array}{l}
\omega_{5}\left[g \sin \left(\varphi_{4}-\varphi_{3}\right) \sin \left(\varphi_{2}-\varphi_{5}-\alpha\right)-e \sin \left(\varphi_{2}-\varphi_{3}\right) \sin \left(\varphi_{4}-\phi_{5}\right)\right] \\
=\left[\left(\dot{x}_{B}-\dot{x}_{C}\right) \cdot \cos \varphi_{2}+\left(\dot{y}_{B}-\dot{y}_{C}\right) \cdot \sin \varphi_{2}\right] \cdot \sin \left(\varphi_{4}-\varphi_{3}\right) \\
-\left[\left(\dot{x}_{D}-\dot{x}_{C}\right) \cdot \cos \varphi_{4}+\left(\dot{y}_{D}-\dot{y}_{C}\right) \cdot \sin \varphi_{4}\right] \cdot \sin \left(\varphi_{2}-\varphi_{3}\right)
\end{array}\right.
$$

The expression (24) is derived in relation to time to obtain the expression of the angular acceleration $\varepsilon_{5}$ directly. Thus, the relation (25) is obtained, from which the value of the angular acceleration $\varepsilon_{5}$ in the form (26) is then explained:

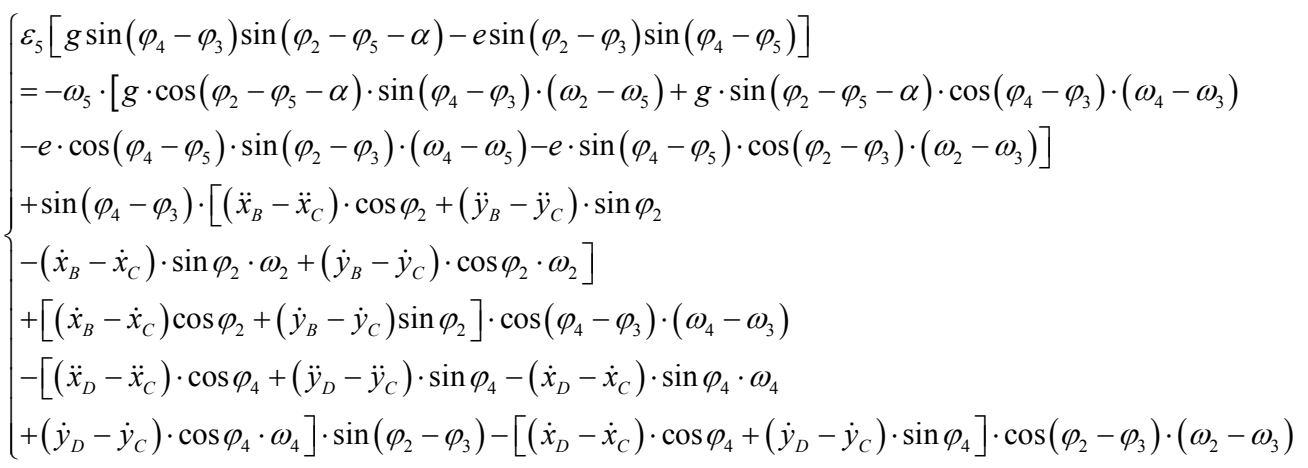

$\varepsilon_{5}=\left\{-\omega_{5} \cdot\left[g \cdot \cos \left(\varphi_{2}-\varphi_{5}-\alpha\right) \cdot \sin \left(\varphi_{4}-\varphi_{3}\right) \cdot\left(\omega_{2}-\omega_{5}\right)\right.\right.$

$+g \cdot \sin \left(\varphi_{2}-\varphi_{5}-\alpha\right) \cdot \cos \left(\varphi_{4}-\varphi_{3}\right) \cdot\left(\omega_{4}-\omega_{3}\right)-e \cdot \cos \left(\varphi_{4}-\varphi_{5}\right) \cdot \sin \left(\varphi_{2}-\varphi_{3}\right) \cdot\left(\omega_{4}-\omega_{5}\right)$

$\left.-e \cdot \sin \left(\varphi_{4}-\varphi_{5}\right) \cdot \cos \left(\varphi_{2}-\varphi_{3}\right) \cdot\left(\omega_{2}-\omega_{3}\right)\right]+\sin \left(\varphi_{4}-\varphi_{3}\right) \cdot\left[\left(\ddot{x}_{B}-\ddot{x}_{C}\right) \cdot \cos \varphi_{2}+\left(\ddot{y}_{B}-\ddot{y}_{C}\right) \cdot \sin \varphi_{2}\right.$

$\left.-\left(\dot{x}_{B}-\dot{x}_{C}\right) \cdot \sin \varphi_{2} \cdot \omega_{2}+\left(\dot{y}_{B}-\dot{y}_{C}\right) \cdot \cos \varphi_{2} \cdot \omega_{2}\right]$

$+\left[\left(\dot{x}_{B}-\dot{x}_{C}\right) \cos \varphi_{2}+\left(\dot{y}_{B}-\dot{y}_{C}\right) \sin \varphi_{2}\right] \cdot \cos \left(\varphi_{4}-\varphi_{3}\right) \cdot\left(\omega_{4}-\omega_{3}\right)$

$\left[\left(\ddot{x}_{D}-\ddot{x}_{C}\right) \cdot \cos \varphi_{4}+\left(\ddot{y}_{D}-\ddot{y}_{C}\right) \cdot \sin \varphi_{4}-\left(\dot{x}_{D}-\dot{x}_{C}\right) \cdot \sin \varphi_{4} \cdot \omega_{4}+\left(\dot{y}_{D}-\dot{y}_{C}\right) \cdot \cos \varphi_{4} \cdot \omega_{4}\right] \cdot \sin \left(\varphi_{2}-\varphi_{3}\right)$

$\left.-\left[\left(\dot{x}_{D}-\dot{x}_{C}\right) \cos \varphi_{4}+\left(\dot{y}_{D}-\dot{y}_{C}\right) \sin \varphi_{4}\right] \cos \left(\varphi_{2}-\varphi_{3}\right)\left(\omega_{2}-\omega_{3}\right)\right\}$

$:\left[g \sin \left(\varphi_{4}-\varphi_{3}\right) \sin \left(\varphi_{2}-\varphi_{5}-\alpha\right)-e \sin \left(\varphi_{2}-\varphi_{3}\right) \sin \left(\varphi_{4}-\varphi_{5}\right)\right]$

The expression (27) of the angular velocity $\omega_{2}$ is further derived and the angular acceleration $\varepsilon_{2}$ (relationship 28) is obtained directly:

$\omega_{2}=\frac{\left(\dot{x}_{B}-\dot{x}_{C}\right) \cdot \sin \varphi_{2}+\left(\dot{y}_{C}-\dot{y}_{B}\right) \cdot \cos \varphi_{2}}{l_{2}}+\frac{l_{3} \cdot \omega_{3} \cdot \cos \left(\varphi_{3}-\varphi_{2}\right)+g \cdot \omega_{5} \cdot \cos \left(\varphi_{2}-\varphi_{5}-\alpha\right)}{l_{2}}$

$\left\{\begin{array}{l}\varepsilon_{2}=\frac{1}{l_{2}} \cdot\left[\left(\ddot{x}_{B}-\ddot{x}_{C}\right) \cdot \sin \varphi_{2}+\left(\ddot{y}_{C}-\ddot{y}_{B}\right) \cdot \cos \varphi_{2}\right. \\ +\left(\dot{x}_{B}-\dot{x}_{C}\right) \cdot \cos \varphi_{2} \cdot \omega_{2}-\left(\dot{y}_{C}-\dot{y}_{B}\right) \cdot \sin \varphi_{2} \cdot \omega_{2} \\ +l_{3} \cdot \varepsilon_{3} \cdot \cos \left(\varphi_{3}-\varphi_{2}\right)-l_{3} \cdot \omega_{3} \cdot \sin \left(\varphi_{3}-\varphi_{2}\right) \cdot\left(\omega_{3}-\omega_{2}\right) \\ \left.+g \cdot \varepsilon_{5} \cdot \cos \left(\varphi_{2}-\varphi_{5}-\alpha\right)-g \cdot \omega_{5} \cdot \sin \left(\varphi_{2}-\varphi_{5}-\alpha\right) \cdot\left(\omega_{2}-\omega_{5}\right)\right]\end{array}\right.$ 
Then the expression (29) of the angular velocity $\omega_{4}$ is derived, and the expression of the angular acceleration $\varepsilon_{4}$ (relationship 30) is obtained:

$$
\begin{aligned}
& \omega_{4}=\frac{\left(\dot{x}_{D}-\dot{x}_{C}\right) \cdot \sin \varphi_{4}+\left(\dot{y}_{C}-\dot{y}_{D}\right) \cdot \cos \varphi_{4}}{l_{4}} \\
& +\frac{l_{3} \cdot \omega_{3} \cdot \cos \left(\varphi_{4}-\varphi_{3}\right)+e \cdot \omega_{5} \cdot \cos \left(\varphi_{4}-\varphi_{5}\right)}{l_{4}}
\end{aligned}
$$

$$
\left\{\begin{array}{l}
\varepsilon_{4}=\frac{1}{l_{4}} \cdot\left[\left(\ddot{x}_{D}-\ddot{x}_{C}\right) \cdot \sin \varphi_{4}+\left(\ddot{y}_{C}-\ddot{y}_{D}\right) \cdot \cos \varphi_{4}\right. \\
+\left(\dot{x}_{D}-\dot{x}_{C}\right) \cdot \cos \varphi_{4} \cdot \omega_{4}-\left(\dot{y}_{C}-\dot{y}_{D}\right) \cdot \sin \varphi_{4} \cdot \omega_{4} \\
+l_{3} \cdot \varepsilon_{3} \cdot \cos \left(\varphi_{4}-\varphi_{3}\right)-l_{3} \cdot \omega_{3} \cdot \sin \left(\varphi_{4}-\varphi_{3}\right) \cdot\left(\omega_{4}-\omega_{3}\right) \\
\left.+e \cdot \varepsilon_{5} \cdot \cos \left(\varphi_{4}-\varphi_{5}\right)-e \cdot \omega_{5} \cdot \sin \left(\varphi_{4}-\varphi_{5}\right) \cdot\left(\omega_{4}-\omega_{5}\right)\right]
\end{array}\right.
$$

\section{Results and Discussion}

The kinematics of inner dome couplings and weight centers on each element of the 6R diaphragm (Fig. 2 and relational systems 31-32) can be further determined:

$$
\begin{aligned}
& \begin{array}{c}
\left\{\begin{array}{l}
\left\{\begin{array} { l } 
{ x _ { E } = x _ { B } + l _ { 2 } \cdot \operatorname { c o s } \varphi _ { 2 } } \\
{ y _ { E } = y _ { B } + l _ { 2 } \cdot \operatorname { s i n } \varphi _ { 2 } }
\end{array} \Rightarrow \left\{\begin{array} { l } 
{ \dot { x } _ { E } = \dot { x } _ { B } - l _ { 2 } \cdot \operatorname { s i n } \varphi _ { 2 } \cdot \omega _ { 2 } } \\
{ \dot { y } _ { E } = \dot { y } _ { B } + l _ { 2 } \cdot \operatorname { c o s } \varphi _ { 2 } \cdot \omega _ { 2 } }
\end{array} \Rightarrow \left\{\begin{array}{l}
\ddot{x}_{E}=\ddot{x}_{B}-l_{2} \cdot \cos \varphi_{2} \cdot \omega_{2}^{2}-l_{2} \cdot \sin \varphi_{2} \cdot \varepsilon_{2} \\
\ddot{y}_{E}=\ddot{y}_{B}-l_{2} \cdot \sin \varphi_{2} \cdot \omega_{2}^{2}+l_{2} \cdot \cos \varphi_{2} \cdot \varepsilon_{2}
\end{array}\right.\right.\right. \\
\left\{\begin{array} { l } 
{ x _ { F } = x _ { C } + l _ { 3 } \cdot \operatorname { c o s } \varphi _ { 3 } } \\
{ y _ { F } = y _ { C } + l _ { 3 } \cdot \operatorname { s i n } \varphi _ { 3 } }
\end{array} \Rightarrow \left\{\begin{array} { l } 
{ \dot { x } _ { F } = \dot { x } _ { C } - l _ { 3 } \cdot \operatorname { s i n } \varphi _ { 3 } \cdot \omega _ { 3 } } \\
{ \dot { y } _ { F } = \dot { y } _ { C } + l _ { 3 } \cdot \operatorname { c o s } \varphi _ { 3 } \cdot \omega _ { 3 } }
\end{array} \Rightarrow \left\{\begin{array}{l}
\ddot{x}_{F}=\ddot{x}_{C}-l_{3} \cdot \cos \varphi_{3} \cdot \omega_{3}^{2}-l_{3} \cdot \sin \varphi_{3} \cdot \varepsilon_{3} \\
\ddot{y}_{F}=\ddot{y}_{C}-l_{3} \cdot \sin \varphi_{3} \cdot \omega_{3}^{2}+l_{3} \cdot \cos \varphi_{3} \cdot \varepsilon_{3}
\end{array}\right.\right.\right.
\end{array}\right. \\
\left\{\begin{array} { l } 
{ x _ { G } = x _ { D } + l _ { 4 } \cdot \operatorname { c o s } \varphi _ { 4 } } \\
{ y _ { G } = y _ { D } + l _ { 4 } \cdot \operatorname { s i n } \varphi _ { 4 } }
\end{array} \Rightarrow \left\{\begin{array} { l } 
{ \dot { x } _ { G } = \dot { x } _ { D } - l _ { 4 } \cdot \operatorname { s i n } \varphi _ { 4 } \cdot \omega _ { 4 } } \\
{ \dot { y } _ { G } = \dot { y } _ { D } + l _ { 4 } \cdot \operatorname { c o s } \varphi _ { 4 } \cdot \omega _ { 4 } }
\end{array} \Rightarrow \left\{\begin{array}{l}
\ddot{x}_{G}=\ddot{x}_{D}-l_{4} \cdot \cos \varphi_{4} \cdot \omega_{4}^{2}-l_{4} \cdot \sin \varphi_{4} \cdot \varepsilon_{4} \\
\ddot{y}_{G}=\ddot{y}_{D}-l_{4} \cdot \sin \varphi_{4} \cdot \omega_{4}^{2}+l_{4} \cdot \cos \varphi_{4} \cdot \varepsilon_{4}
\end{array}\right.\right.\right.
\end{array}
\end{aligned}
$$

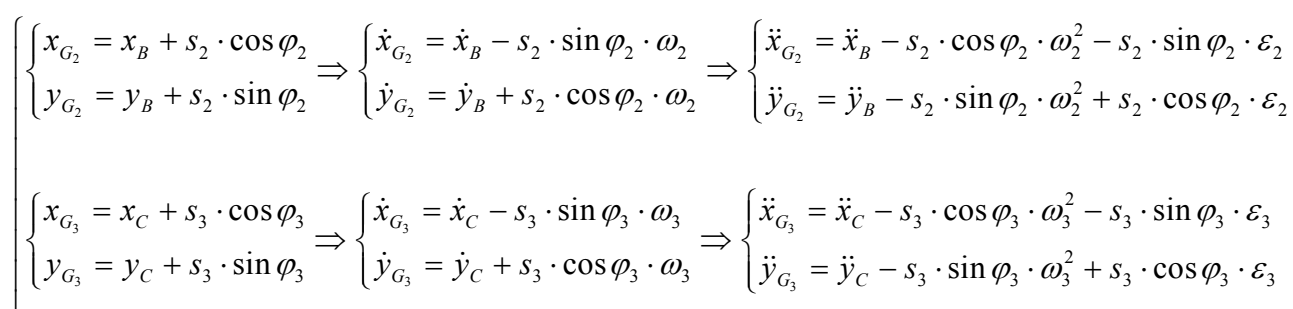

$$
\begin{aligned}
& \left\{\begin{array} { l } 
{ x _ { G _ { 4 } } = x _ { D } + s _ { 4 } \cdot \operatorname { c o s } \varphi _ { 4 } } \\
{ y _ { G _ { 4 } } = y _ { D } + s _ { 4 } \cdot \operatorname { s i n } \varphi _ { 4 } }
\end{array} \Rightarrow \left\{\begin{array} { l } 
{ \dot { x } _ { G _ { 4 } } = \dot { x } _ { D } - s _ { 4 } \cdot \operatorname { s i n } \varphi _ { 4 } \cdot \omega _ { 4 } } \\
{ \dot { y } _ { G _ { 4 } } = \dot { y } _ { D } + s _ { 4 } \cdot \operatorname { c o s } \varphi _ { 4 } \cdot \omega _ { 4 } }
\end{array} \Rightarrow \left\{\begin{array}{l}
\ddot{x}_{G_{4}}=\ddot{x}_{D}-s_{4} \cdot \cos \varphi_{4} \cdot \omega_{4}^{2}-s_{4} \cdot \sin \varphi_{4} \cdot \varepsilon_{4} \\
\ddot{y}_{G_{4}}=\ddot{y}_{D}-s_{4} \cdot \sin \varphi_{4} \cdot \omega_{4}^{2}+s_{4} \cdot \cos \varphi_{4} \cdot \varepsilon_{4}
\end{array}\right.\right.\right. \\
& \left\{\begin{array} { l } 
{ x _ { G _ { 5 } } = x _ { F } + s _ { 5 } \cdot \operatorname { c o s } ( \varphi _ { 5 } + \beta ) } \\
{ y _ { G _ { 5 } } = y _ { F } + s _ { 5 } \cdot \operatorname { s i n } ( \varphi _ { 5 } + \beta ) }
\end{array} \Rightarrow \left\{\begin{array}{l}
\dot{x}_{G_{5}}=\dot{x}_{F}-s_{5} \cdot \sin \left(\varphi_{5}+\beta\right) \cdot \omega_{5} \\
\dot{y}_{G_{5}}=\dot{y}_{F}+s_{5} \cdot \cos \left(\varphi_{5}+\beta\right) \cdot \omega_{5}
\end{array}\right.\right. \\
& \Rightarrow\left\{\begin{array}{l}
\ddot{x}_{G_{5}}=\ddot{x}_{F}-s_{5} \cdot \cos \left(\varphi_{5}+\beta\right) \cdot \omega_{5}^{2}-s_{5} \cdot \sin \left(\varphi_{5}+\beta\right) \cdot \varepsilon_{5} \\
\ddot{y}_{G_{5}}=\ddot{y}_{F}-s_{5} \cdot \sin \left(\varphi_{5}+\beta\right) \cdot \omega_{5}^{2}+s_{5} \cdot \cos \left(\varphi_{5}+\beta\right) \cdot \varepsilon_{5}
\end{array}\right.
\end{aligned}
$$

With the help of the kinematics of the center of gravity, in the future, it is possible to determine the triad kinetostatic and its dynamics.

Such groups have applications in mechanics, mechanisms, robots, thermal engines, aircraft. 


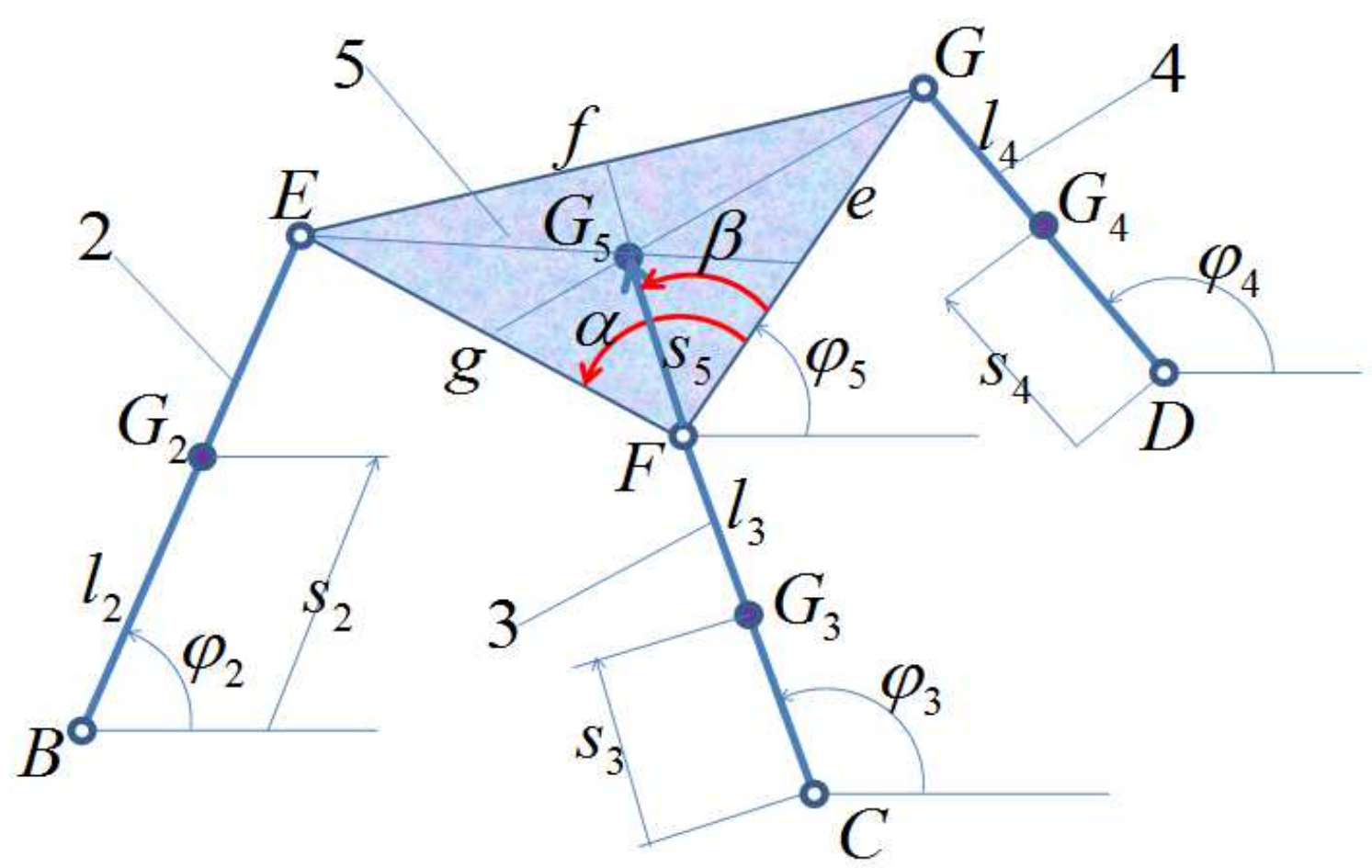

Fig. 2: The kinematics of gravity centers at a $6 \mathrm{R}$ triad

\section{Conclusion}

The present work is intended presenting a triad kinematics general used only with the kinematic couplings rotational (C5), because such approaches are rare in the area, although triad is a structured group Assuric often used. The calculation theoretical method presented is an analytical one.

Such groups have applications in mechanics, mechanisms, robots, thermal engines, aircraft.

\section{Acknowledgement}

This text was acknowledged and appreciated by Dr. Veturia CHIROIU Honorific member of Technical Sciences Academy of Romania (ASTR) PhD supervisor in Mechanical Engineering.

\section{Author's Contributions}

All the authors contributed equally to prepare, develop and carry out this manuscript.

\section{Ethics}

This article is original and contains unpublished material. Authors declare that are not ethical issues and no conflict of interest that may arise after the publication of this manuscript.

\section{References}

Antonescu, P., 2000. Mechanisms and Handlers, Printech Publishing House. Bucharest.

Antonescu, P. and F. Petrescu, 1985. Analytical method of synthesis of cam mechanism and flat stick. Proceedings of the 4th International Symposium on Mechanism Theory and Practice (TPM' 85), Bucharest.

Antonescu, P. and F. Petrescu, 1989. Contributions to cinetoelastodynamic analysis of distribution mechanisms. Bucharest.

Antonescu, P., M. Oprean and F. Petrescu, 1985a. Contributions to the synthesis of oscillating cam mechanism and oscillating flat stick. Proceedings of the 4th International Symposium on Theory and Practice of Mechanisms, (TPM' 85), Bucharest.

Antonescu, P., M. Oprean and F. Petrescu, 1985b. At the projection of the oscillante cams, there are mechanisms and distribution variables. Proceedings of the V-Conference for Engines, Automobiles, Tractors and Agricultural Machines, I-Engines and Automobiles, (AMA’ 85), Brasov.

Antonescu, P., M. Oprean and F. Petrescu, 1986. Projection of the profile of the rotating camshaft acting on the oscillating plate with disengagement. Proceedings of the 3rd National Computer Assisted Designing Symposium in Mechanisms and Machine Bodies, (MOM' 86), Brasov. 
Antonescu, P., M. Oprean and F. Petrescu, 1987. Dynamic analysis of the cam distribution mechanisms. Proceedings of the Seventh National Symposium of Industrial Robots and Spatial Mechanisms (IMS' 87), Bucharest,

Antonescu, P., M. Oprean and F. Petrescu, 1988. Analytical synthesis of Kurz profile, rotating flat cam cam. Machine Build. Rev. Bucharest.

Antonescu, P., F. Petrescu and O. Antonescu, 1994. Contributions to the synthesis of the rotating cam mechanism and the tip of the balancing tip. Brasov.

Antonescu, P., F. Petrescu and D. Antonescu, 1997. Geometrical synthesis of the rotary cam and balance tappet mechanism. Bucharest.

Antonescu, P., F. Petrescu and O. Antonescu, 2000a. Contributions to the synthesis of the rotary disc-cam profile. Proceedings of the 8th International Conference on Theory of Machines and Mechanisms, (TMM' 00), Liberec, Czech Republic, pp: 51-56.

Antonescu, P., F. Petrescu and O. Antonescu, 2000b. Synthesis of the rotary cam profile with balance follower. Proceedings of the 8th Symposium on Mechanisms and Mechanical Transmissions (MMT' 000), Timişoara, pp: 39-44.

Antonescu, P., F. Petrescu and O. Antonescu, 2001. Contributions to the synthesis of mechanisms with rotary disc-cam. Proceedings of the 8th IFToMM International Symposium on Theory of Machines and Mechanisms, (TMM' 01), Bucharest, ROMANIA, pp: 31-36.

Aversa, R., R.V. Petrescu, A. Apicella and F.I.T. Petrescu, 2017a. Nano-diamond hybrid materials for structural biomedical application. Am. J. Biochem. Biotechnol., 13: 34-41. DOI: 10.3844/ajbbsp.2017.34.41

Aversa, R., R.V. Petrescu, B. Akash, R.B. Bucinell and J.M. Corchado et al., 2017b. Kinematics and forces to a new model forging manipulator. Am. J. Applied Sci., 14: 60-80. DOI: 10.3844/ajassp.2017.60.80

Aversa, R., R.V. Petrescu, A. Apicella, F.I.T. Petrescu and J.K. Calautit et al., 2017c. Something about the $\mathrm{V}$ engines design. Am. J. Applied Sci., 14: 34-52. DOI: 10.3844/ajassp.2017.34.52

Aversa, R., D. Parcesepe, R.V. Petrescu, F. Berto and G. Chen et al., 2017d. Processability of bulk metallic glasses. Am. J. Applied Sci., 14: 294-301. DOI: 10.3844/ajassp.2017.294.301

Aversa, R., R.V. Petrescu, A. Apicella and F.I.T. Petrescu, 2017e. Modern transportation and photovoltaic energy for urban ecotourism. Transylvanian Rev. Administrative Sci., 13: 5-20. DOI: $10.24193 /$ tras.SI2017.1

Aversa, R., F.I.T. Petrescu, R.V. Petrescu and A. Apicella, 2016a. Biomimetic FEA bone modeling for customized hybrid biological prostheses development. Am. J. Applied Sci., 13: 1060-1067. DOI: 10.3844/ajassp.2016.1060.1067
Aversa, R., D. Parcesepe, R.V. Petrescu, G. Chen and F.I.T. Petrescu et al., 2016b. Glassy amorphous metal injection molded induced morphological defects. Am. J. Applied Sci., 13: 1476-1482. DOI: 10.3844 /ajassp.2016.1476.1482

Aversa, R., R.V. Petrescu, F.I.T. Petrescu and A. Apicella, 2016c. Smart-factory: Optimization and process control of composite centrifuged pipes. Am. J. Applied Sci., 13: 1330-1341.

DOI: 10.3844/ajassp.2016.1330.1341

Aversa, R., F. Tamburrino, R.V. Petrescu, F.I.T. Petrescu and M. Artur et al., $2016 \mathrm{~d}$. Biomechanically inspired shape memory effect machines driven by muscle like acting NiTi alloys. Am. J. Applied Sci., 13: 1264-1271.

DOI: 10.3844/ajassp.2016.1264.1271

Cao, W., H. Ding, Z. Bin and C. Ziming, 2013. New structural representation and digital-analysis platform for symmetrical parallel mechanisms. Int. J. Adv. Robot. Sys. DOI: 10.5772/56380

Comănescu, A., D. Comănescu, I. Dugăeșescu and A. Boureci, 2010. The Basics of Modeling Mechanisms. 1st Edn., Politehnica Press Publishing House, Bucharest, ISBN-10: 978-606-515-115-4, pp: 274.

Dong, H., N. Giakoumidis, N. Figueroa and N. Mavridis, 2013. Approaching behaviour monitor and vibration indication in developing a General Moving Object Alarm System (GMOAS). Int. J. Adv. Robot. Sys. DOI: $10.5772 / 56586$

De Melo, L.F., R.A., S.F. Rosário and J.M., Rosário, 2012. Mobile robot navigation modelling, control and applications. Int. Rev. Modell. Simulations, 5: 1059-1068.

Garcia, E., M.A. Jimenez, P.G. De Santos and M. Armada, 2007. The evolution of robotics research. IEEE Robot. Autom. Magaz., 14: 90-103. DOI: 10.1109/MRA.2007.339608

Garcia-Murillo, M., J. Gallardo-Alvarado and E. Castillo-Castaneda, 2013. Finding the generalized forces of a series-parallel manipulator. IJARS. DOI: $10.5772 / 53824$

He, B., Z. Wang, Q. Li, H. Xie and R. Shen, 2013. An analytic method for the kinematics and dynamics of a multiple-backbone continuum robot. IJARS. DOI: $10.5772 / 54051$

Lee, B.J., 2013. Geometrical derivation of differential kinematics to calibrate model parameters of flexible manipulator. Int. J. Adv. Robot. Sys. DOI: $10.5772 / 55592$

Lin, W., B. Li, X. Yang and D. Zhang, 2013. Modelling and control of inverse dynamics for a 5-DOF parallel kinematic polishing machine. Int. J. Adv. Robot. Sys. DOI: $10.5772 / 54966$

Liu, H., W. Zhou, X. Lai and S. Zhu, 2013. An efficient inverse kinematic algorithm for a PUMA560structured robot manipulator. IJARS.

DOI: $10.5772 / 56403$ 
Mirsayar, M.M., V.A. Joneidi, R.V. Petrescu, F.I.T. Petrescu and F. Berto, 2017. Extended MTSN criterion for fracture analysis of soda lime glass. Eng. Fracture Mechan., 178: 50-59.

DOI: 10.1016/j.engfracmech.2017.04.018

Padula, F. and V. Perdereau, 2013. An on-line path planner for industrial manipulators. Int. J. Adv. Robot. Sys. DOI: 10.5772/55063

Pelecudi, C., 1967. The Basics of Mechanism Analysis. Publishing house: Academy of the People's Republic of Romania.

Perumaal, S. and N. Jawahar, 2013. Automated trajectory planner of industrial robot for pick-andplace task. IJARS. DOI: 10.5772/53940

Petrescu, F. and R. Petrescu, 1995a. Contributions to optimization of the polynomial motion laws of the stick from the internal combustion engine distribution mechanism. Bucharest.

Petrescu, F. and R. Petrescu, 1995b. Contributions to the synthesis of internal combustion engine distribution mechanisms. Bucharest.

Petrescu, F. and R. Petrescu, 1997a. Dynamics of cam mechanisms (exemplified on the classic distribution mechanism). Bucharest.

Petrescu, F. and R. Petrescu, 1997b. Contributions to the synthesis of the distribution mechanisms of internal combustion engines with Cartesian coordinate method. Bucharest.

Petrescu, F. and R. Petrescu, 1997c. Contributions to maximizing polynomial laws for the active stroke of the distribution mechanism from internal combustion engines. Bucharest.

Petrescu, F. and R. Petrescu, 2000a. Synthesis of distribution mechanisms by the rectangular (cartesian) coordinate method. University of Craiova, Craiova.

Petrescu, F. and R. Petrescu, 2000b. The design (synthesis) of cams using the polar coordinate method (the triangle method). University of Craiova, Craiova.

Petrescu, F. and R. Petrescu, 2002a. Motion laws for cams. Proceedings of the 7th National Symposium with International Participation Computer Assisted Design (PAC' 02), Braşov, pp: 321-326.

Petrescu, F. and R. Petrescu, 2002b. Camshaft dynamics elements. Proceedings of the 7th National Symposium with International Participation Computer Assisted Design (PAC' 02), Braşov, pp: 327-332.

Petrescu, F. and R. Petrescu, 2003. Some elements regarding the improvement of the engine design. Proceedings of the 8th National Symposium, Descriptive Geometry, Technical Graphics and Design, (GTD’03), Braşov, pp: 353-358.
Petrescu, F. and R. Petrescu, 2005a. The cam design for a better efficiency. Proceedings of the International Conference on Engineering Graphics and Design, (EGD’ 05), Bucharest, pp: 245-248.

Petrescu, F. and R. Petrescu, 2005b. Contributions at the dynamics of cams. Proceedings of the 9th IFToMM International Symposium on Theory of Machines and Mechanisms, (TMM' 05), Bucharest, Romania, pp: 123-128.

Petrescu, F. and R. Petrescu, 2005c. Determining the dynamic efficiency of cams. Proceedings of the 9th IFToMM International Symposium on Theory of Machines and Mechanisms, (TMM' 05), Bucharest, Romania, pp: 129-134.

Petrescu, F. and R. Petrescu, 2005d. An original internal combustion engine. Proceedings of the 9th IFToMM International Symposium on Theory of Machines and Mechanisms, (TMM' 05), Bucharest, Romania, pp: $135-140$.

Petrescu, F. and R. Petrescu, 2005e. Determining the mechanical efficiency of Otto engine's mechanism. Proceedings of the 9th IFToMM International Symposium on Theory of Machines and Mechanisms, (TMM' 05), Bucharest, Romania, pp: 141-146.

Petrescu, F.I. and R.V. Petrescu, 2013. Cinematics of the 3R Dyad. Engevista, 15: 118-124.

Petrescu, F.I. and R.V. Petrescu, 2012a. Kinematics of the planar quadrilateral mechanism. Engevista, 14: 345-348.

Petrescu, F.I. and R.V. Petrescu, 2012b. MecatronicaSisteme Seriale si Paralele. Create Space Publisher, USA, ISBN-10: 978-1-4750-6613-5, pp: 128.

Petrescu, F.I. and R.V. Petrescu, 2011. Mechanical Systems, Serial and Parallel-Course (in Romanian). LULU Publisher, London, UK, ISBN-10: 978-1-4466-0039-9, pp: 124.

Petrescu, F.I. and R.V. Petrescu, 2016a. Parallel moving mechanical systems kinematics, ENGEVISTA, 18: 455-491.

Petrescu, F.I. and R.V. Petrescu, 2016b. Direct and inverse kinematics to the Anthropomorphic Robots, ENGEVISTA, 18: 109-124.

Petrescu, F. and R. Petrescu, 2016c. An otto engine dynamic model. IJM\&P, 7: 038-048.

Petrescu, F.I. and R.V. Petrescu, 2016d. Otto motor dynamics, GEINTEC, 6: 3392-3406.

Petrescu, F.I. and R.V. Petrescu, 2016e. Dynamic cinematic to a structure 2R. GEINTEC, 6: 3143-3154.

Petrescu, F.I., B. Grecu, A. Comanescu and R.V. Petrescu, 2009. Some mechanical design elements. Proceeding of the International Conference on Computational Mechanics and Virtual Engineering, (MEC’ 09), Braşov, pp: 520-525.

Petrescu, R.V., R. Aversa, A. Apicella, M.M. Mirsayar and F.I.T. Petrescu, 2016a About the gear efficiency to a simple planetary train. Am. J. Applied Sci., 13: 1428-1436. 
Petrescu, R.V., R. Aversa, A. Apicella, S. Li and G. Chen et al., 2016b. Something about electron dimension. Am. J. Applied Sci., 13: 1272-1276.

Petrescu, F.I.T., A. Apicella, R. Aversa, R.V. Petrescu and J.K. Calautit et al., 2016c. Something about the mechanical moment of inertia. Am. J. Applied Sci., 13: 1085-1090.

Petrescu, R.V., R. Aversa, A. Apicella, F. Berto and S. Li et al., 2016d. Ecosphere protection through green energy. Am. J. Applied Sci., 13: 1027-1032.

Petrescu, F.I.T., A. Apicella, R.V. Petrescu, S.P. Kozaitis and R.B. Bucinell et al., 2016e. Environmental protection through nuclear energy. Am. J. Applied Sci., 13: 941-946.

Petrescu, F.I.T. and J.K. Calautit, 2016a. About nano fusion and dynamic fusion. Am. J. Applied Sci., 13: 261-266.

Petrescu, F.I.T. and J.K. Calautit, 2016b. About the light dimensions. Am. J. Applied Sci., 13: 321-325.

Petrescu, R.V., R. Aversa, B. Akash, R. Bucinell and J. Corchado et al., 2017a. Modern propulsions for aerospace-a review. J. Aircraft Spacecraft Technol., 1: 1-8. DOI: $10.3844 /$ jastsp.2017.1.8

Petrescu, R.V., R. Aversa, B. Akash, R. Bucinell and J. Corchado et al., 2017b. Modern propulsions for aerospace-part II. J. Aircraft Spacecraft Technol., 1: 9-17. DOI: 10.3844/jastsp.2017.9.17

Petrescu, R.V., R. Aversa, B. Akash, R. Bucinell and J. Corchado et al., 2017c. History of aviation-a short review. J. Aircraft Spacecraft Technol., 1: 30-49. DOI: 10.3844 /jastsp.2017.30.49

Petrescu, R.V., R. Aversa, B. Akash, R. Bucinell and J. Corchado et al., 2017d. Lockheed martin-a short review. J. Aircraft Spacecraft Technol., 1: 50-68. DOI: 10.3844 jastsp.2017.50.68

Petrescu, R.V., R. Aversa, B. Akash, J. Corchado and F. Berto et al., 2017e. Our universe. J. Aircraft Spacecraft Technol., 1: 69-79. DOI: 10.3844/jastsp.2017.69.79

Petrescu, R.V., R. Aversa, B. Akash, J. Corchado and F. Berto et al., 2017f. What is a UFO? J. Aircraft Spacecraft Technol., 1: 80-90.

DOI: 10.3844 jastsp.2017.80.90

Petrescu, R.V., R. Aversa, B. Akash, J. Corchado and F. Berto et al., 2017g. About bell helicopter FCX-001 concept aircraft-a short review. J. Aircraft Spacecraft Technol., 1: 91-96.

DOI: 10.3844 jastsp.2017.91.96

Petrescu, R.V., R. Aversa, B. Akash, J. Corchado and F. Berto et al., 2017h. Home at airbus. J. Aircraft Spacecraft Technol., 1: 97-118.

DOI: 10.3844 jastsp.2017.97.118

Petrescu, R.V., R. Aversa, B. Akash, J. Corchado and F. Berto et al., 2017i. Airlander. J. Aircraft Spacecraft Technol., 1: 119-148.

DOI: $10.3844 /$ jastsp.2017.119.148
Petrescu, R.V., R. Aversa, B. Akash, J. Corchado and F. Berto et al., 2017j. When boeing is dreaming-a review. J. Aircraft Spacecraft Technol., 1: 149-161. DOI: $10.3844 /$ jastsp.2017.149.161

Petrescu, R.V., R. Aversa, B. Akash, J. Corchado and F. Berto et al., 2017k. About Northrop Grumman. J. Aircraft Spacecraft Technol., 1: 162-185. DOI: $10.3844 /$ jastsp.2017.162.185

Petrescu, R.V., R. Aversa, B. Akash, J. Corchado and F. Berto et al., 20171. Some special aircraft. J. Aircraft Spacecraft Technol., 1: 186-203. DOI: $10.3844 /$ jastsp.2017.186.203

Petrescu, R.V., R. Aversa, B. Akash, J. Corchado and F. Berto et al., 2017m. About helicopters. J. Aircraft Spacecraft Technol., 1: 204-223. DOI: $10.3844 /$ jastsp.2017.204.223

Petrescu, R.V., R. Aversa, B. Akash, F. Berto and A. Apicella et al., 2017n. The modern flight. J. Aircraft Spacecraft Technol., 1: 224-233. DOI: 10.3844 /jastsp.2017.224.233

Petrescu, R.V., R. Aversa, B. Akash, F. Berto and A. Apicella et al., 2017o. Sustainable energy for aerospace vessels. J. Aircraft Spacecraft Technol., 1: 234-240. DOI: 10.3844/jastsp.2017.234.240

Petrescu, R.V., R. Aversa, B. Akash, F. Berto and A. Apicella et al., 2017p. Unmanned helicopters. J. Aircraft Spacecraft Technol., 1: 241-248. DOI: $10.3844 /$ jastsp.2017.241.248

Petrescu, R.V., R. Aversa, B. Akash, F. Berto and A. Apicella et al., 2017q. Project HARP. J. Aircraft Spacecraft Technol., 1: 249-257. DOI: $10.3844 /$ jastsp.2017.249.257

Petrescu, R.V., R. Aversa, B. Akash, F. Berto and A. Apicella et al., 2017r. Presentation of romanian engineers who contributed to the development of global aeronautics-part I. J. Aircraft Spacecraft Technol., 1: 258-271. DOI: $10.3844 /$ jastsp.2017.258.271

Petrescu, R.V., R. Aversa, B. Akash, F. Berto and A. Apicella et al., 2017s. A first-class ticket to the planet mars, please. J. Aircraft Spacecraft Technol., 1: 272-281. DOI: 10.3844/jastsp.2017.272.281

Petrescu, R.V., R. Aversa, B. Akash, F. Berto and A. Apicella et al., 2017t. Forces of a 3R robot. J. Mechatronics Robotics, 1: 1-14. DOI: $10.3844 /$ jmrsp.2017.1.14

Petrescu, R.V., R. Aversa, B. Akash, F. Berto and A. Apicella et al., 2017u. Direct geometry and cinematic to the MP-3R systems. J. Mechatronics Robotics, 1: 15-23. DOI: 10.3844/jmrsp.2017.15.23

Petrescu, R.V., R. Aversa, B. Akash, F. Berto and A. Apicella et al., 2017v. Dynamic elements at MP3R. J. Mechatronics Robotics, 1: 24-37. DOI: 10.3844/jmrsp.2017.24.37 
Petrescu, R.V., R. Aversa, B. Akash, F. Berto and A. Apicella et al., 2017w. Geometry and direct kinematics to MP3R with $4 \times 4$ operators. J. Mechatronics Robotics, 1: 38-46. DOI: $10.3844 /$ jmrsp.2017.38.46

Petrescu, R.V., R. Aversa, A. Apicella, M.M. Mirsayar and S. Kozaitis et al., 2017x. Current stage in the field of mechanisms with gears and rods. J. Mechatronics Robotics, 1: 47-57. DOI: $10.3844 /$ jmrsp.2017.47.57

Petrescu, R.V., R. Aversa, A. Apicella, M.M. Mirsayar and S. Kozaitis et al., 2017y. Geometry and inverse kinematic at the MP3R mobile systems. J. Mechatronics Robotics, 1: 58-65. DOI: $10.3844 / \mathrm{jmrsp} .2017 .58 .65$

Petrescu, R.V., R. Aversa, A. Apicella, M.M. Mirsayar and S. Kozaitis et al., 2017z. Synthesis of optimal trajectories with functions control at the level of the kinematic drive couplings. J. Mechatronics Robotics, 1: 66-74. DOI: 10.3844/jmrsp.2017.66.74

Petrescu, R.V., R. Aversa, A. Apicella, M.M. Mirsayar and S. Kozaitis et al., 2017aa. The inverse kinematics of the plane system 2-3 in a mechatronic MP2R system, by a trigonometric method. J. Mechatronics Robotics, 1: 75-87. DOI: $10.3844 /$ jmrsp.2017.75.87

Petrescu, R.V., R. Aversa, A. Apicella, M.M. Mirsayar and S. Kozaitis et al., 2017ab. Serial, anthropomorphic, spatial, mechatronic systems can be studied more simply in a plan. J. Mechatronics Robotics, 1: 88-97. DOI: 10.3844/jmrsp.2017.88.97

Petrescu, R.V., R. Aversa, A. Apicella, M.M. Mirsayar and S. Kozaitis et al., 2017ac. Analysis and synthesis of mechanisms with bars and gears used in robots and manipulators. J. Mechatronics Robotics, 1: 98-108. DOI: 10.3844/jmrsp.2017.98.108
Petrescu, R.V., R. Aversa, A. Apicella, M.M. Mirsayar and S. Kozaitis et al., 2017ad. Speeds and accelerations in direct kinematics to the MP3R systems. J. Mechatronics Robotics, 1: 109-117. DOI: $10.3844 /$ jmrsp.2017.109.117

Petrescu, R.V., R. Aversa, A. Apicella, M.M. Mirsayar and S. Kozaitis et al., 2017ae. Geometry and determining the positions of a plan transporter manipulator. J. Mechatronics Robotics, 1: 118-126. DOI: $10.3844 /$ jmrsp.2017.118.126

Reddy, P., K.V. Shihabudheen and J. Jacob, 2012. Precise non linear modeling of flexible link flexible joint manipulator. IReMoS, 5: 1368-1374.

Tabaković, S., M. Zeljković, R. Gatalo and A. Živković, 2013. Program suite for conceptual designing of parallel mechanism-based robots and machine tools. Int. J. Adv. Robot Sys.

DOI: $10.5772 / 56633$

Tang, X., D. Sun and Z. Shao, 2013. The structure and dimensional design of a reconfigurable PKM. IJARS. DOI: $10.5772 / 54696$

Tong, G., J. Gu and W. Xie, 2013. Virtual entity-based rapid prototype for design and simulation of humanoid robots. Int. J. Adv. Robot. Sys. DOI: $10.5772 / 55936$

Wang, K., M. Luo, T. Mei, J. Zhao and Y. Cao, 2013. Dynamics analysis of a three-DOF planar serialparallel mechanism for active dynamic balancing with respect to a given trajectory. Int. J. Adv. Robotic Sys. DOI: 10.5772/54201

Wen, S., J. Zhu, X. Li, A. Rad and X. Chen, 2012. Endpoint contact force control with quantitative feedback theory for mobile robots. IJARS.

DOI: $10.5772 / 53742$ 